\title{
Note sur la place des mots
}

Note on the Place of Words

\section{Antonia García Castro}

\section{Q OpenEdition}

1 Journals

\section{Édition électronique}

URL : http://journals.openedition.org/conflits/19591

DOI : 10.4000/conflits. 19591

ISSN : $1777-5345$

Éditeur :

CCLS - Centre d'études sur les conflits lilberté et sécurité, L'Harmattan

\section{Édition imprimée}

Date de publication : 30 octobre 2017

Pagination : 158-163

ISBN : 978-2-343-13540-3

ISSN : 1157-996X

\section{Référence électronique}

Antonia García Castro, « Note sur la place des mots », Cultures \& Conflits [En ligne], 107 | automne 2017, mis en ligne le 30 octobre 2017, consulté le 30 mars 2021. URL : http://journals.openedition.org/ conflits/19591; DOI : https://doi.org/10.4000/conflits.19591 


\section{Note sur la place des mots}

\section{Antonia GARCIA CASTRO}

Antonia García Castro est docteure en sociologie, chercheuse associée à l'ISP, responsable de la rubrique Regards sur l'entre-deux de Cultures \& Conflits depuis 2005, traductrice spécialisée en sciences sociales et littérature.

$\bigcup_{*}$ qui suit est une note à la marge. En lien avec le texte d'Eduardo Rinesi, «Il n’y a aucune possibilité d'entente entre nous ». Le politologue et philosophe argentin y poursuit ses réflexions sur les tragédies shakespeariennes et, plus particulièrement, sur Hamlet ${ }^{1}$. Ici revisitée du point de vue de la langue, ce qui inclut à la fois les problèmes de langue - de compréhension tels qu'ils se posent entre les personnages de la pièce, et les difficultés que soulèvent les traductions de la pièce de l'anglais vers l'espagnol et de l'anglais vers le français. L'une des questions que Rinesi pose, et qui lui permet de penser conjointement traduction et politique, est celle de la décision. Tout comme l'acteur politique - nous dit l'auteur -, tout comme Hamlet en personne, le traducteur est parfois amené à faire des choix lourds de conséquences. Et ce rapprochement qui peut sembler cocasse, qui sans doute l'est, est aussi une proposition tout à fait sérieuse qui tourne autour du mot («choix »), puis le retourne dans divers sens afin de voir ce qui s'y joue.

On peut ainsi noter, en marge, ou dans la prolongation de ces réflexions, quelques dilemmes propres à la traduction de ce texte, mais aussi quelques considérations sur la fonction ou plutôt sur le rôle du traducteur. Car, s'il est vrai que le terme renvoie aujourd'hui à un métier qu'on peut apprendre, comme n'importe quel métier, en suivant une formation, la traduction reste aussi une activité que tel ou tel (mais probablement pas n'importe qui ou plutôt pas tout un chacun) peut assumer dans une conjoncture particulière. C'est un fait, outre les écoles spécialisées, il existe encore de nos jours une école de traduction qui est une école de la rue, des expériences qu'on a eues; et aussi des destins, des parcours de traducteurs qui sont à eux seuls une école ${ }^{2}$.

1. Eduardo Rinesi a consacré plusieurs ouvrages à ces questions dont Las máscaras de Jano. Notas sobre el drama de la historia, Buenos Aires, editorial Gorla, 2014. Pour le sujet qui nous occupe ici, il importe de signaler qu'il est aussi l'auteur d'une traduction de Hamlet (Los Polvorines, ediciones UNGS, 2017). 
Les enfants de l'immigration le savent bien puisque, très souvent, ils sont les premiers à apprendre la langue du pays où ils se rendent, et deviennent les traducteurs de leurs parents, de leurs aînés, dans des circonstances plus ou moins (extra)ordinaires, selon le contexte des immigrations. Le politique est bien présent dans ces situations où le besoin de comprendre et de se faire comprendre peut se réveler crucial pour l'avenir d'une famille, même s'il est plus manfestement présent dans d'autres contextes : on conçoit aisément l'importance du rôle du traducteur sur la grande scène des échanges internationaux entre présidents de la République, par exemple. Dans la même optique, ce n'est sans doute pas par hasard que, en Amérique latine, l'une des figures de la Conquête, figure légendaire, s'occupe de traduction : Malinche, interprète d'Hernán Cortès, devenue un symbole de la trahison aux peuples autochtones.

Mais, par rapport aux questions qui intéressent Rinesi, notamment lorsqu'il aborde à la fin de son texte cette « sensation primordiale - dit-il - que nous éprouvons à chaque fois que notre chemin bifurque dans deux directions différentes ", il me semble qu'on en trouve une illustration particulièrement intéressante dans un tout autre contexte, celui de la seconde guerre mondiale. Il s'agit du film de Roberto Benigni, La vita è bella (1997). Lorsque le personnage incarné par Benigni s'improvise traducteur dans un camp de concentration où il est enfermé avec son petit garçon de cinq ans. C'est une scène remarquable où non seulement ce qui est le plus souvent présenté comme la raison d'être de toute traduction (comprendre, se comprendre) n'est plus le but du jeu... mais où l'enjeu est précisément l'inverse : il ne faut surtout pas que le petit garcon comprenne ce que dit l'autorité allemande. Il faut même, et c'est une question de survie, que le petit comprenne tout à fait autre chose. En l'occurrence, qu'il soit assuré que son père dit vrai lorsqu'il soutient que tout ce qu'ils sont en train de vivre dans ce camp n'est qu'un jeu. Benigni (Guido, dans le film) se lance alors. Poussé par le besoin, il joue le traducteur, travestit, déguise le discours du gardien du camp, pour rester fidèle à son rôle de père protecteur de cet enfant qu'il chérit.

Il est intéressant de noter que, pour que cette fausse traduction reste tout de même crédible, Benigni reproduit le ton employé ainsi que les mimiques. Et, sans doute, on ne traduit pas que des mots. Ou, plutôt, les mots impliquent toujours autre chose que des mots. Ni les définitions, telles qu'elles apparaissent dans les dictionnaires, ni les usages toujours mouvants, ne sont en soi suffisants, puisque les mots sont aussi incarnés, porteurs d'une intention, d'un souffle et, parfois, d'une gestuelle.

2. C'est notamment le cas de la traductrice de Dostoïevski en langue allemande, Svetlana Geier. Son parcours, ainsi que sa façon particulière de concevoir la traduction ont fait l'objet d'un film important pour quiconque s'intéresse à ce qu'engage le métier de traducteur. Voir $\mathrm{La}$ femme aux 5 éléphants, un film de Vadim Jendreyko, 2009. 
C'est, en tous cas, ce qui arrive dans le domaine des traductions littéraires ou, plus généralement, dans le domaine des traductions qui nous mettent en présence d'un auteur. Et c'est aussi ce qui fait que le dispositif qu'on va élaborer pour procéder à une traduction peut être à revoir, à repenser, voire à réinventer, à recommencer, pour une autre.

S'agissant de la traduction du texte de Rinesi («Il n’y a aucune possibilité d'entente entre nous»), les choix ont été nombreux et ils ne sont pas tellement racontables. Ils ne le sont pas, dans ce sens où une traduction, avant même de mettre en place une sorte de pacte entre traducteur et lecteur, engage un pacte entre traducteur et auteur. Notamment parce que le traducteur (ici la traductrice) est tout de même persuadé(e) du contraire : une forme d'entente entre nous reste possible. On se comprend. Et c'est parce qu'on se comprend, qu'on peut éprouver le souhait de partager un texte dans une autre langue, auprès d'un autre public. De ce point de vue, on peut noter en passant qu'un certain optimisme est attaché à la condition de traducteur. Et même, si en lecteur assidu de Rinesi, ce traducteur comprend qu'il est - c'est juste - parfaitement impossible de tout rendre, il n'en reste pas moins qu'il peut toujours choisir ce qu'il ne conçoit pas de ne pas rendre. Ce à quoi il ne saurait renoncer sans que la traduction ne perde sa raison d'être ${ }^{3}$.

Dans le cas qui nous occupe, il y avait un certain nombre de défis qui étaient aussi des motivations. Il s'agissait de traduire un texte qui portait sur la traduction. Plus précisément, il s'agissait de traduire en français un texte écrit par un auteur argentin, en partie consacré aux traductions de Hamlet, de l'anglais vers l'espagnol, mais aussi - c'est là que la chose se corsait... - de l'anglais vers le français puisque l'auteur s'intéressait aux réflexions que Derrida avait consacrées à ces questions des traductions shakespeariennes ${ }^{4}$. La difficulté tenait donc au fait que ce travail de traduction mettait en présence non pas deux mais trois langues. Elle tenait aussi au fait qu'il n'y avait pas, dans ce cas, de sens - pourrait-on dire - préétabli. Quelle serait ici la langue de départ ? La langue d'arrivée?

Le chemin à faire n'était pas droit mais circulaire. Ou plutôt, il s'agissait de faire une série d'allers-retours. Là où l'auteur, en amont de son texte, avait d'abord dû regarder les traductions de l'anglais vers l'espagnol, avant de se tourner vers le francais. En aval, pour être à même de présenter ce texte au lecteur français, il me fallait renverser cette priorité et regarder d'abord les traductions de l'anglais vers le français, avant d'éventuellement me pencher sur

3. C'est particulièrement vrai lorsque le traducteur soutient son texte de bout en bout, c'est-àdire lorsqu'il est non seulement l'exécutant mais le porteur d'un projet de traduction qu'il va présenter à telle ou telle maison d'édition, revue, etc.

4. Derrida J., Spectres de Marx. L'État de la dette, le travail du denil et la nouvelle Internationale, Paris, éditions Galilée, 1993. 
les traductions de l'anglais vers l'espagnol. Non seulement parce que l'auteur citait en espagnol une série de répliques de Hamlet qu'il fallait reproduire en français, mais aussi parce que, par moments, il ne citait pas. Il ne citait pas, mais incorporait directement à son propos des passages, des références à des répliques de la pièce.

Ainsi, une partie du travail a consisté à lister non seulement les citations mais toute référence, et à comparer les différentes traductions françaises auxquelles j'avais accès en Argentine, et qui n'étaient que trois ${ }^{5}$. Le but de ce travail n'était pas - du moins, en première instance - de déterminer quelle était la traduction française la plus proche de l'anglais... mais quelle était la traduction française la plus proche de la traduction en espagnol utilisée par l'auteur. Car c'est bien d'abord à mon auteur et à sa lecture de Hamlet que ma traduction entendait rester fidèle - autant que faire se peut - et non pas directement à l'œuvre commentée. Ce qui était d'autant plus vrai que, dans le cas de ce texte, Rinesi a eu recours à sa propre traduction publiée en 2017.

Au cours de ce travail de lecture des diverses traductions françaises, mises en regard avec une traduction en espagnol, nous avons pu remarquer (l'auteur et moi-même) toutes sortes de situations. Parfois, les difficultés - mais aussi les possibilités de restitution - semblaient proches en français et en espagnol. Parfois, elles étaient différentes. Certains choix nous ont aussi laissés perplexes. L'une des choses qui a le plus attiré mon attention, c'est la disparition ici et là de certains mots.

En effet, à un certain moment l'auteur nous dit :

Ainsi, lorsque le spectre du père de Hamlet s'en va en lui donnant l'ordre de se rappeler de lui, Hamlet reste seul sur scène et assume cette promesse "while memory holds a seat in this distracted globe " $(1.5 .96-7)$ : «tant que vivra la mémoire sur notre globe détraqué », en jouant avec le triple, et non plus le double sens de « globe».

La traduction utilisée ici est celle de Derocquigny. C’est celle qui semble le mieux rendre l'expression que Rinesi va analyser dans ce paragraphe. Le problème se pose, quelques lignes plus loin, lorsque l'auteur reprend cette réplique pour insister cette fois-ci sur l'importance d'un autre mot : seat. Le mot ne figure pas dans la traduction de Derocquigny. Il ne figure pas non plus dans celle de Gide, qui choisit : « aussi longtemps que mémoire habitera ce monde affolé ». François-Victor Hugo avait opté pour « tant que ma mémoire aura son siège dans ce globe égaré », plus proche de l'original concernant les

5. Hamlet, trad. Derocquigny J., Paris, J.-M. Dent \& Fils, 1922 ; trad. André Gide, Paris, Gallimard, 1946 ; trad. François-Victor Hugo, Euvres complètes de Shakespeare, Paris, Pagnerre, [1865], 1872. 
deux mots commentés par Rinesi (mais pourquoi «ma » mémoire, là où il n’y avait que «mémoire» ?). Le fait est que Rinesi poursuit son analyse et que, si on ne disposait que des deux premières traductions, sans accès à l'original, sa lecture ne serait pas compréhensible.

En effet, le nom du théâtre de Shakespeare, The Globe, constitue une référence évidente à cette vision baroque du monde comme théâtre, de la vie comme représentation, qui est un thème récurrent dans toute l'œuvre de Shakespeare et, particulièrement, dans cette pièce. C'est pour cela que l'emploi, par Hamlet, du mot seat (« while memory holds a seat... ») ${ }^{6}$ a du sens. Parce que seat c'est la place... dans un théâtre. La mémoire de l'ancien roi doit garder sa place, nous dit son fils, un siège, un espace dans ce théâtre qu'est le monde, dans sa tête détraquée, et dans ce théâtre même où tout cela est en train de se dire sur une scène.

On saisit bien ici aussi tout ce qui peut se jouer non plus seulement dans le fait de choisir de rendre tel mot par tel autre, dans une autre langue, mais le danger qu'il peut y avoir à prendre trop au sérieux, ou trop à cœur l'idée que j'énonçais plus haut : on ne traduit pas que des mots. Car il arrive aussi que chaque mot compte, que chaque mot cuente : dans le sens que ce mot prend en espagnol, de contar (compter et raconter). Comme un excès de méfiance vis-àvis de la traduction mot à mot qui aurait pour effet de faire disparaitre, sans le vouloir, une toute petite pièce du récit qui pourrait s'avérer fondamentale.

Pour finir cette note à la marge, j’aimerais signaler ce à quoi - pour reprendre une des expressions de l'auteur - je préférais ne pas renoncer en tant que traductrice de ce texte. Il n'y avait pas qu'une seule chose, évidemment. Mais il y avait surtout une chose, qui résume bien toutes les autres. Cela concerne le dernier paragraphe.

Pero me gustaría aventurar, sin haber sido nunca príncipe, $y$ habiendo sido solo unas pocas veces $-y$ de puro irresponsable- traductor, que no debe ser menos angustiante que ella, ni menos conocedora de esa sensación primordial que experimentamos cada vez que nuestro camino se abre en dos direcciones diferentes y solo tenemos como consejeros a nuestra prudencia, nuestro buen sentido, nuestra sabiduría para aceptar las pérdidas que nos imponga nuestra decisión y nuestra determinación para, seguros de una sola cosa: de que fracasaremos, avanzar.

6. Ce qui en espagnol est rendu par « mientras la memoria tenga un sitio en este globo trastornado». Trad. Eduardo Rinesi, op. cit. 
Mais j'aimerais aventurer, sans avoir jamais été prince et ayant été quelquefois traducteur - par pure irresponsabilité -, qu'il n'en est pas moins angoissant, ni moins porteur de la sensation primordiale que nous éprouvons à chaque fois que notre chemin bifurque dans deux directions différentes et que nous avons comme seuls conseillers notre prudence, notre bon sens, notre sagesse pour accepter les pertes que nous impose notre décision, et notre détermination (alors même que nous sommes sûrs de l'échec) pour avancer.

Au fil des lectures réalisées, des propositions de correction ont été apportées. La version ici présentée en tient compte. Il y avait néanmoins une suggestion qui revenait d'une lecture à l'autre et qui me semblait difficile à accepter. Cela concernait l'ordre des mots en toute fin de paragraphe. Je savais bien que mes lecteurs avaient raison du point de vue de la langue. En français, cette phrase sonnait mieux, passait mieux, en modifiant l'ordre des mots. D'ailleurs, c'était une phrase impossible. Moi-même, en espagnol, j'avais butté. La structure en était quelque peu baroque et on pouvait penser à une erreur. J'étais donc devant ce texte, d'abord en lectrice qui cherche à comprendre un texte écrit par un auteur qui s'exprime en espagnol, et qui s'exprime ici, en Argentine, en ce moment - je veux dire en ce moment précis de l'Argentine, mais aussi en ce moment précis du monde. Pour finir, il m'a fallu lire à voix haute. Ce n'est que là que j'ai compris. J'ai compris que je ne faisais pas la pause au bon endroit, qu'il y avait une difficulté liée à la respiration (ce qu'une des lectrices a ensuite soulignée dans ma traduction qui, pourtant, avait conscience de cette pause... sans se décider à l'indiquer : "il manque là une virgule, on ne comprend pas sans cette virgule »). Le fait est qu'une fois que cette phrase s'est révélée compréhensible non pas à mes yeux mais plutôt à mes oreilles, la chose fut une évidence : il n'était pas concevable de changer l'ordre des mots. Il fallait absolument respecter leur place. Car, si je procédais au déplacement, le dernier mot de ce texte qui portait sur les décisions, sur les choix qui se présentent à nous dans certaines circonstances particulièrement difficiles, et qu'on ne maîtrise jamais complètement, serait « échec ». Or, le dernier mot de Rinesi était avanzar. Avancer. 
